\title{
Obtención de compuesto de titanio con gradiente de porosidad mediante técnica pulvimetalúrgica
}

\author{
Luciano Grinschpun ${ }^{1}$, Carlos Oldani ${ }^{1}$, Matías Schneiter ${ }^{1,2}$, \\ Matías Valdemarin ${ }^{1}$, Juan Pereyra ${ }^{1}$
}

\author{
${ }^{1}$ Departamento de Materiales y Tecnología, Facultad de Ciencias Exactas, Físicas y Naturales, Universidad Nacional de \\ Córdoba, Av. Vélez Sarsfield 1611, Córdoba, Argentina. \\ ${ }^{2}$ Instituto de Astronomía Teórica y Experimental-CONICET, Laprida 854, Córdoba, Argentina. \\ e-mail: lgrin.cvmt@gmail.com
}

\section{RESUMEN}

En el estudio de biomateriales de uso implantológico para la fabricación de distintos tipos de prótesis, el titanio es un metal que ha sido utilizado exitosamente debido a su buena resistencia a la corrosión y a su buena resistencia mecánica. A pesar de los avances realizados, en la actualidad aún subsisten algunos problemas con este material en aplicaciones de reemplazo óseo debido al alto valor de módulo elástico de este metal $(110 \mathrm{GPa})$ en relación al del hueso cortical (20-30 GPa) y al hueso trabecular (1-5 GPa). Esta diferencia en los módulos elásticos del titanio y le hueso es una de las principales causas de falla del proceso de implantación de prótesis óseas. El fallo se debe al fenómeno de apantallamiento de tensiones (stress shielding) que genera una disminución en la densidad ósea (osteopenia) debilitando el hueso que eventualmente termina por romperse. Una de las técnicas utilizadas para disminuir el módulo elástico del titanio consiste en la fabricación de un compuesto poroso mediante técnicas pulvimetalúrgicas, sinterizando una mezcla de polvos de titanio y agentes espaciadores que se eliminan durante el tratamiento térmico. Por las características estructurales de los huesos, es lógico pensar en la posibilidad de fabricar prótesis con gradiente de porosidad, replicando de alguna manera, la estructura del mismo. Otra de las problemáticas que se presentan en el uso de titanio para implantes es la incapacidad del mismo en promover los procesos biológicos de osteointegración. Existen distintas propuestas para disminuir este problema. Una es la incorporación de hidroxiapatita (HA) con el fin de promover los fenómenos de osteointegración. Sin embargo, la hidroxiapatita(HA) se descompone en presencia de titanio a las temperaturas normales de sinterización de este metal, degradando el compuesto en poco tiempo. En este trabajo se exponen los resultados obtenidos en la fabricación de muestras de titanio con gradiente de porosidad radial a partir del sinterizado de una mezcla de hidruro de titanio y bicarbonato de amonio. Para la obtención de las muestras se estudiaron distintas técnicas de fabricación analizando las distintas etapas en la técnica pulvimetalurgica (mezcla de polvos, distintos métodos de prensado para la fabricación de modelos en verdes y tratamientos térmicos de sinterizado). El material obtenido fue caracterizado mecánicamente mediante ensayo de compresión para determinar su módulo de elasticidad. En las muestras ensayadas con gradiente de porosidad radial se obtuvieron valores de módulo elástico de 16,4 a 20 GPa. Así mismo se realizó la caracterización física de la porosidad con distintos métodos (análisis metalográfico y métodos gravimétricos). Los resultados indican que las muestras con porosidad radial tienen una porosidad promedio del $18 \%$. Los datos obtenidos se utilizaron para estimar el módulo de elasticidad del compuesto y comparar estos resultados con datos empíricos obtenidos mediante ensayos de compresión.

Palabras clave: titanio, biomaterial, poroso, radial.

\section{ABSTRACT}

Within the study of biomaterials for implantological use titanium is among the most widely employed metals due to its excellent corrosion and mechanical resistance. In spite of the advances made, there are some issues remaining with the value of the elastic moduli of the titanium $(110 \mathrm{GPa})$ in comparison with the elastic moduli of the cortical bone (20-30 GPa) and the trabecular bone (1-5GPa). This difference is one of the main reasons a bone prosthesis fails once implanted, producing what is known as Stress shielding. One of the techniques employed to diminish the elastic moduli of titanium consists in incorporating pores to the material. 
This is done through powder metallurgy techniques, allowing the fabrications of porous composites, sintering a mix of powders of metallic hydrides and spacers that are eliminated during the heat treatment. Taking into account the structural characteristics of the bone, it makes sense to think on the possibility of fabricating a prosthesis with pore gradients. Another problem encountered when employing titanium, is its inability to promote the osteointegration. There are different techniques to tackle this problem, and one of them is to incorporate hydroxyapatite which has the problem that it decomposes in the presence of titanium when sintered at high temperatures. The current study shows the results obtained during the fabrication of samples of titanium with pore gradients. We studied different fabrication techniques, analyzing each stage during the powder metallurgy. process. The obtained material was characterized mechanically through compression tests, determining the elastic moduli of the composite, which was found to be between 16.4 and $20 \mathrm{GPa}$. Also, different physical characterization methods where employed to analyse the pore content which was found to be $18 \%$. The obtained data was employed for the estimation of elastic moduli of the compound, and compared to the empirical results obtained with the mechanical tests.

Keywords: titanium, biomaterial, porous, radial.

\section{INTRODUCCIÓN}

Una breve historia de los biomateriales [1] muestra que las investigaciones destinadas a desarrollar materiales y/o componentes para remplazo de partes del cuerpo humano tienen como denominador común, el diseñar elementos que imiten las propiedades y funciones de los órganos que se pretende reemplazar (propiedades biológicas, físicas, y químicas). El titanio como material de remplazo óseo posee propiedades que lo hacen apto para este fin; buena resistencia mecánica, además su buena resistencia a la corrosión, lo hace un material bioinerte, propiedades estas que lo han convertido en un metal de uso muy extendido en implantología ósea. Por otro lado, pese a las bondades que presenta el titanio como biomaterial, sus propiedades no son óptimas si se lo compara con el hueso. Uno de los problemas que se presenta es el alto valor del módulo elástico en relación al del hueso [2-4]. Esta diferencia trae como consecuencia el fenómeno de apantallamiento de tensiones (stress shielding). Por otro lado, la bioinercia respecto al titanio, hace de este un material que no promueve los procesos biológicos que integran y reparan los huesos. Esta particularidad es causa de falla de los procesos de implantación de prótesis de titanio debido al encapsulamiento fibroso [5-6]. Las problemáticas mencionadas apuntan el camino para desarrollar las investigaciones que mejoren el desempeño del titanio como biomaterial.

Una técnica conocida para disminuir el módulo elástico de un material denso consiste en la incorporación de poros al seno del material para generar un compuesto cuyo modulo elástico sea menor al del material base, debido a la disminución de la sección resistente cuando se consideran secciones nominales iguales. Así mismo una técnica extendida para favorecer los fenómenos de osteointegración en implantes es la incorporación de agentes bioactivos como la hidroxiapatita (HA) al compuesto. Distintos autores han trabajado en el problema de la incompatibilidad mecánica entre titanio y hueso. Oldani y Padilla [3,4] obtuvieron buenos resultados al fabricar compuestos porosos de titanio mediante técnica pulvimetalurgica a partir de polvo de hidruro de titanio $\left(\mathrm{TiH}_{2}\right)$ utilizando bicarbonato de amonio $\left(\mathrm{NH}_{4} \mathrm{HCO}_{3}\right)$ como agente espaciador. Torres et al. [7] obtuvieron resultados similares al probar con otros métodos para generar poros al fabricar compuestos de titanio incorporando además gradiente de porosidad longitudinal. En los casos mencionados, se obtuvieron compuestos porosos de titanio con un módulo elástico inferior al del titanio denso. Es muy variada la cantidad de compuestos bioactivos que se podrían incorporar al compuesto de titanio para modificar su bioinercia. Uno de los más utilizados es la hidroxiapatita (HA) componente cerámico inorgánico principal de hueso [8$11]$.

Integrar en un proceso de fabricación, técnicas que permitan obtener un compuesto de titanio poroso, que además contenga HA, plantea algunos problemas constructivos para las muestras. Gallo y Canavocio [12] señalan los problemas de sinterizar muestras a partir de un mezcla de polvo de titanio e hidroxiapatita (HA) a temperaturas por encima de $\operatorname{los} 1000^{\circ} \mathrm{C}$. Estos autores indican que las muestras obtenidas presentaban desgranamiento al final del tratamiento térmico, o se desgranaban a los pocos días. Distintos autores [1316] han estudiado el proceso de descomposición de la HA a alta temperatura, indicando que el problema se debe a la alta reactividad del titanio y el calcio. Durante el sinterizado a temperaturas superiores a los 1000 ${ }^{\circ} \mathrm{C}$ se forman titanatos de calcio $\left(\mathrm{CaTiO}_{3}\right)$ y compuestos de titanio y fosforo $\left(\mathrm{Ti}_{\mathrm{x}} \mathrm{P}_{\mathrm{y}}\right)$ que se depositan en las partículas de titanio dando como resultado muestras con baja resistencia a la compresión.

En este trabajo se propone estudiar distintas técnicas de fabricación por sinterizado de un compuesto en base a titanio con gradiente de porosidad radial para disminuir el módulo de elasticidad del compuesto. Con el objetivo de incorporar hidroxiapatita (HA) como agente bioactivo a las muestras se estudiaron distin- 
tos tratamientos térmicos para determinar cuánto es posible bajar la temperatura de sinterizado en la fabricación de muestras hechas a partir de polvo de $\mathrm{Ti}_{2}$.

\section{MATERIALES Y MÉTODOS}

En este trabajo se realizaron distintas experiencias tendientes a establecer los pasos necesarios para la fabricación de muestras sinterizadas a partir de polvo de hidruro de titanio $\left(\mathrm{TiH}_{2}\right)$ con gradiente de porosidad radial. En todos los casos las muestras se fabricaron a partir de $\mathrm{TiH}_{2}$ (SIGMA-ALDRICH, -325 mesh). Para las muestras porosas se usó una mezcla de $\mathrm{TiH}_{2}$ y bicarbonato de amonio $\left(\mathrm{NH}_{4} \mathrm{HCO}_{3}\right)$ en distintas proporciones en peso. El $\mathrm{NH}_{4} \mathrm{HCO}_{3}$ se secó previo a la mezcla en desecador de vació durante 60 minutos. Los distintos grados de porosidad para las muestras se consiguieron fraccionando el agente espaciador, midiendo el peso correspondiente a cada volumen de porosidad deseado según el tipo de muestra. Para tener una distribución homogénea de los distintos componentes se utilizó un mezclador rotativo en Y (de vidrio) durante $30 \mathrm{~min}$. La compactación de los verdes se llevó a cabo utilizando matrices metálicas cilíndricas uniaxiales de doble y simple efecto, de diámetros 6,8 y $12 \mathrm{~mm}$. Para la fabricación de las distintas muestras se usó un rango de presiones que va de 40 a $390 \mathrm{MPa}$. Las muestras fueron sinterizadas en horno eléctrico con cámara tubular de acero inoxidable con entrada y salida de gases. Como atmosfera protectora se utilizó gas Argón 5.0. En el sinterizado se siguieron distintos ciclos de tratamiento térmico según el tipo de probeta fabricada en cada caso (descrito más adelante). Las probetas fueron calentadas junto con el horno y, una vez terminado el tratamiento térmico, las muestras fueron enfriadas manteniendo la atmosfera protectora dentro de la cámara hasta una temperatura inferior a $\operatorname{los} 100{ }^{\circ} \mathrm{C}$ para prevenir procesos de oxidación en el Ti. A las muestras obtenidas se les realizó caracterización física para medir porosidad volumétrica según norma ASTM C373-88, estudio metalográfico y caracterización mecánica mediante ensayos de compresión y dureza.

\subsection{Determinación de tiempo mínimo de sinterizado en función de la temperatura}

Uno de los objetivos del presente trabajo es minimizar la temperatura de sinterizado con el fin de incorporar, en un futuro, hidroxiapatita, evitando la degradación del compuesto Ti-Ha en el proceso. Para esto, se sinterizaron muestras fabricadas con $\mathrm{TiH}_{2}$ partiendo de una temperatura de $1000^{\circ} \mathrm{C}$ y un tiempo de 1 hora, luego, buscando determinar la menor temperatura a la que se podían sinterizar muestras de $\mathrm{TiH}_{2}$, se siguió el proceso iterativo resumido en la figura (1). Es decir, se sinterizó con un tiempo y temperatura $\left(\mathrm{t}_{\mathrm{i}}, \mathrm{T}_{\mathrm{i}}\right)$, luego se preparó muestra metalográfica seguida de la medición de dureza en distintos puntos de la sección transversal con el fin de determinar la continuidad estructural de la muestra. Si la variación de la dureza era mínima se fijaba el tiempo de sinterizado para esa temperatura. De lo contrario se procedía a repetir los pasos mencionados para tiempos mayores. La tabla (1) muestra las temperaturas, tiempos y durezas determinados.

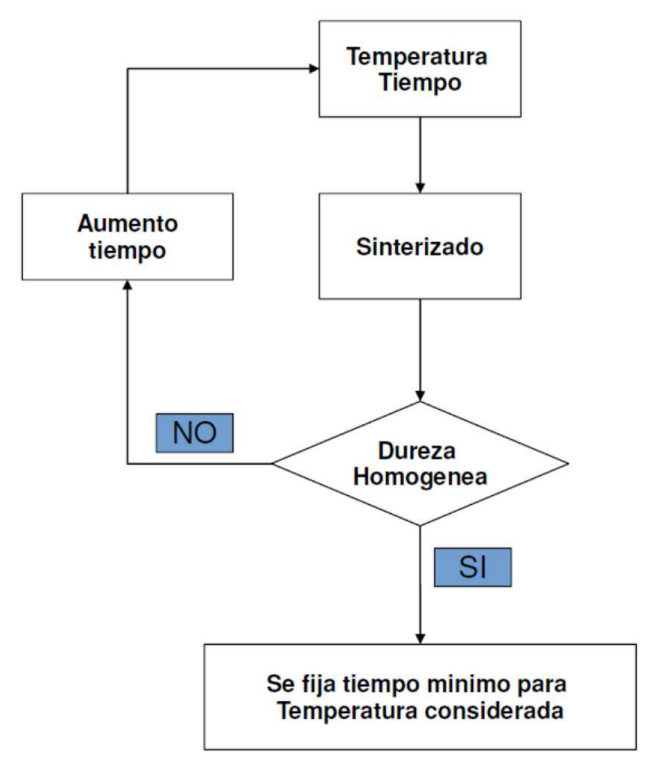

Figura 1: Esquema de tratamientos térmicos. 
Tabla 1: Tiempos mínimos de sinterizado en función de valores medios de dureza obtenidos en cada tratamiento

\begin{tabular}{l|l|l}
\hline $\begin{array}{l}\text { TEMPERATURA DE } \\
\text { SINTERIZADO }\left[{ }^{\circ} \mathbf{C}\right]\end{array}$ & $\begin{array}{l}\text { TIEMPO MINIMO DE } \\
\text { SINTERIZADO }[\mathbf{H}]\end{array}$ & HV (PROMEDIO) \\
\hline 1000 & 1 & 396 \\
\hline 800 & 2 & 215 \\
\hline 600 & 3 & 127 \\
\hline
\end{tabular}

\subsection{Método de obtención de gradientes de porosidad}

El producir un material con un gradiente de porosidad tiene dos finalidades. Por un lado, reducir el módulo elástico del material a valores comparables a los del hueso cortical. Por otro lado, la existencia de poros, dependiendo de las formas, tamaños y distribuciones, promueve la adherencia celular. Por esta razón en este trabajo se plantea el estudio del proceso de fabricación de un material con gradiente poroso, tal que el módulo elástico medio sea semejante al del hueso cortical, donde el núcleo (para el caso de gradiente radial) sea el que cumple la función mecánica y la parte periférica favorezca la colonización celular. El producir un material con un perfil de porosidad plantea muchas dificultades, razón por la cual en este trabajo se optó por fabricar las muestras en partes discretas con distintos porcentajes de porosidad. Esto hace que existan diversas interfaces interactuantes, con posibles expansiones térmicas diferenciadas. Por esta razón, planteamos el estudio del gradiente de porosidad en dos etapas, una con gradientes longitudinales (2.2.1), donde el énfasis está en el estudio de las interfaces, y otra etapa donde se intenta encontrar un principio de mecanismo de producción de gradiente de porosidad radial (2.2.2).

\subsubsection{Porosidad longitudinal}

Para la fabricación de la probeta con gradiente de porosidad longitudinal, se dispusieron en la matriz de compactado, capas con distintos porcentajes en peso de $\mathrm{TiH}_{2}$ y mezcla de $\mathrm{TiH}_{2}$ y $\mathrm{NH}_{4} \mathrm{HCO}_{3}$. Cada capa se fraccionó con un peso total de $0,25 \mathrm{~g}$ de material con los porcentajes indicados en la columna izquierda de la Tabla 2. En el sinterizado, el ciclo del tratamiento térmico seguido fue: $30 \mathrm{~min}$ a $200{ }^{\circ} \mathrm{C}$, ciclo sublimación de $\mathrm{NH}_{4} \mathrm{HCO}_{3} .30 \mathrm{~min}$ a $500^{\circ} \mathrm{C}$ (deshidruración), 90 min a $1000{ }^{\circ} \mathrm{C}$, ciclo de sinterizado. En la columna derecha de la Tabla 2 se muestra una de las muestras fabricadas con gradiente longitudinal de porosidad, donde a simple vista se puede observar una buena unión entre los distintos estratos.

Tabla 2: Composición de cada capa en muestra con porosidad longitudinal y corte transversal de muestra fabricada.

\begin{tabular}{l|l}
\hline $\begin{array}{l}\text { COMPOSICION DE CADA CAPA } \\
\text { (DE ABAJO HACIA ARRIBA) }\end{array}$ & IMAGEN CORTE LONGITUDINAL \\
\hline $\mathrm{Capa} 1$. & \\
$40 \% \mathrm{TiH}_{2}-60 \% \mathrm{NH}_{4} \mathrm{HCO}_{3}$ & \\
\hline $\mathrm{Capa} 2$. & \\
$60 \% \mathrm{TiH}_{2}-40 \% \mathrm{NH}_{4} \mathrm{HCO}_{3}$ & \\
$\mathrm{Capa} 3:$ & \\
$80 \% \mathrm{TiH}_{2}-20 \% \mathrm{NH}_{4} \mathrm{HCO}_{3}$ & \\
$\mathrm{Capa} 4:$ & \\
$100 \% \mathrm{TiH}_{2}$ & \\
\hline
\end{tabular}

\subsubsection{Porosidad radial}

El gradiente de porosidad radial en las muestras se consiguió a partir de controlar la distribución y mezclas de polvos $\mathrm{TiH}_{2}$ y $\mathrm{TiH}_{2}+\mathrm{NH}_{4} \mathrm{HCO}_{3}$, durante el llenado de las matrices de compactado. Se usaron matrices cilíndricas metálicas de diámetro creciente $(6,8$ y $12 \mathrm{~mm})$ de simple y doble efecto.

El procedimiento general consistió en aplicar individualmente la presión de compactación en la muestra a medida que se agregan las sucesivas capas. Primero se compactó el centro de la probeta en la matriz diámetro $6 \mathrm{~mm}$ usando solo $\mathrm{TiH}_{2}$. El verde obtenido se colocó centrado en la matriz de diámetro $8 \mathrm{~mm}$ donde 
se añadió mezcla de polvos $\mathrm{TiH}_{2}+\mathrm{NH}_{4} \mathrm{HCO}_{3}$ hasta enrasar con el verde de $6 \mathrm{~mm}$ de diámetro. La mezcla de polvo se preparó para obtener una capa con $20 \%$ de porosidad y se aplicó presión para densificar el verde, repitiendo el procedimiento para la tercera capa en la cual se agregó una cantidad de mezcla de polvos tal, que diera como resultado un $40 \%$ porosidad en la capa. Para la primera probeta se utilizó una presión de compactación de $390 \mathrm{MPa}$ en cada etapa, pero se observó falta de unión entre las capas del verde. Por esta razón se decidió bajar la presión de compactación a un mínimo que permitiera obtener un verde manipulable. Distintas experiencias permitieron fijar una presión de compactación mínima de 40 MPa para los verdes fabricados con las matrices diámetros 6 y $8 \mathrm{~mm}$, y 140 MPa para el verde final de diámetro 12mm. En Tabla 3 se indican la composición de las capas y corte transversal de una de las muestras obtenidas donde se observa el gradiente de porosidad.

Tabla 3: Composición de cada capa en muestra con porosidad radial y corte transversal de muestra fabricada.

\begin{tabular}{|c|c|}
\hline $\begin{array}{l}\text { COMPOSICION DE CADA } \\
\text { CAPA (DE IZQUIERDA A } \\
\text { DERECHA) }\end{array}$ & IMAGEN CORTE RADIAL \\
\hline $\begin{array}{l}\text { Capa } 1 . \\
100 \% \mathrm{TiH}_{2}\end{array}$ & 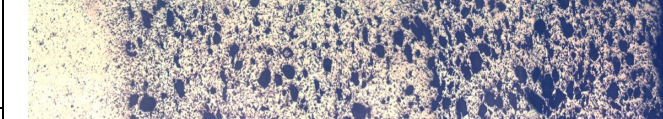 \\
\hline $\begin{array}{l}\text { Capa 2: } \\
80 \% \mathrm{TiH}_{2}-20 \% \mathrm{NH}_{4} \mathrm{HCO}_{3}\end{array}$ & row \\
\hline $\begin{array}{l}\text { Capa 3: } \\
60 \% \mathrm{TiH}_{2}-40 \% \mathrm{NH}_{4} \mathrm{HCO}_{3}\end{array}$ & \\
\hline
\end{tabular}

Se fabricaron además muestras con $20 \%$ y $40 \%$ de porosidad. Presión de compactación: $140 \mathrm{MPa}$, las que fueron caracterizadas mecánicamente mediante ensayo de compresión para tener datos comparativos que permitieran sacar conclusiones ajustadas a los parámetros de presión de compactación que posibilitaron la fabricación del verde con gradiente de porosidad radial. El ciclo térmico seguido en la fabricación de las muestras fue: $30 \mathrm{~min} .200{ }^{\circ} \mathrm{C}$ (sublimación de $\mathrm{NH}_{4} \mathrm{HCO}_{3}$ ), $30 \mathrm{~min} .500{ }^{\circ} \mathrm{C}$ (deshidratación), $180 \mathrm{~min} .800{ }^{\circ} \mathrm{C}$ (sinterizado). En la Tabla 4 se muestran los valores de porosidad del grupo de probetas fabricadas con una presión de compactación de $140 \mathrm{MPa}$

Tabla 4: Porosidad en muestras.

\begin{tabular}{l|l}
\hline $\begin{array}{l}\text { MUESTRA. } \\
\text { COMPOSICION DE CADA CAPA }\end{array}$ & $\%$ POROSIDAD \\
\hline $\mathrm{TiH}_{2}$ & 12 \\
\hline $\mathrm{Ti} \mathrm{H}_{2}+20 \% \mathrm{NH}_{4} \mathrm{HCO}_{3}$ & $30-32$ \\
\hline $\mathrm{Ti} \mathrm{H}_{2}+40 \% \mathrm{NH}_{4} \mathrm{HCO}_{3}$ & $53-55,5$ \\
\hline Muestra con tres capas radiales & $17-18$ \\
\hline
\end{tabular}

\section{RESULTADOS Y DISCUSIÓN}

\subsection{La porosidad y su efecto en las propiedades mecánicas}

En materiales porosos preparados para ser utilizados como reemplazo óseo, las características del poro son un factor que afecta de manera directa a las propiedades mecánicas y biológicas. En los procesos pulvimetalurgicos existen dos tipos de poros, los inherentes al proceso de fabricación de las piezas, poros que son difíciles de controlar y dependen de las granulometrías y distribuciones granulométricas del polvo del material utilizado [17] así como del tratamiento térmico y poros producidos de manera forzada a partir del uso de separadores (bicarbonato de amonio en este caso) para poder controlar la cantidad y el tamaño de los poros. Medir la porosidad en la pieza final no es una tarea fácil y existen diversas técnicas. En el presente trabajo la medición se llevó a cabo a partir de la técnica de Arquímedes según la Norma ASTM C 373-88. Para mode- 
lar el efecto que tienen los poros en las propiedades mecánicas se utilizó la relación de Nielsen (1) [18], la cual vincula el módulo elástico $\left(\mathrm{E}_{\mathrm{N}}\right)$ con la cantidad de poros $(\mathrm{P})$, el módulo elástico de titanio (Eti), y el factor de forma de los poros $\left(\mathrm{F}_{\mathrm{f}}\right)$, de la siguiente manera:

$$
E_{N}=E_{T i}\left[\frac{(1-P / 100)^{2}}{1+\left(\frac{1}{F_{f}}-1\right) 100}\right]
$$

La Figura 2 muestra el módulos elásticos $\mathrm{E}_{\mathrm{N}} \mathrm{y}$ el módulo elástico medido $\mathrm{E}_{\mathrm{E}}$ mediante ensayo de compresión donde se puede observar un descenso del módulo elástico a medida que crece la porosidad. A la izquierda del gráfico la separación entre los dos resultados es más grande. Torres et. al. [19] indican que la medición del módulo de Young a partir de ensayos uniaxiales en ensayos de compresión da resultados significativamente menores que los medidos dinámicamente. Estos autores encontraron que medir la porosidad con ultrasonido da mejor resultado para porosidades pequeñas, lo cual puede explicar la discrepancia creciente hacia las porosidades bajas. . El estudio metalográfico reveló que la porosidad radial (figura 5) pasa de interconectada en la periferia de la muestra a cerrada en la capa intermedia y en el centro solo se observa porosidad intrínseca del sinterizado. La discrepancia entre $\mathrm{E}_{\mathrm{N}}$ y $\mathrm{E}_{\mathrm{E}}$ podría atribuirse también a la variabilidad en la porosidad de las muestras con gradiente radial dado que los gradientes y el cambio de tipo de porosidad en la sección transversal de la muestra (figura 4) dificultan la cuantificación de la porosidad en estas muestras y de esta manera no permiten hacer una valoración del modo en que las distintas capas en las muestras con porosidad radial contribuyen a la resistencia mecánica de la muestra.

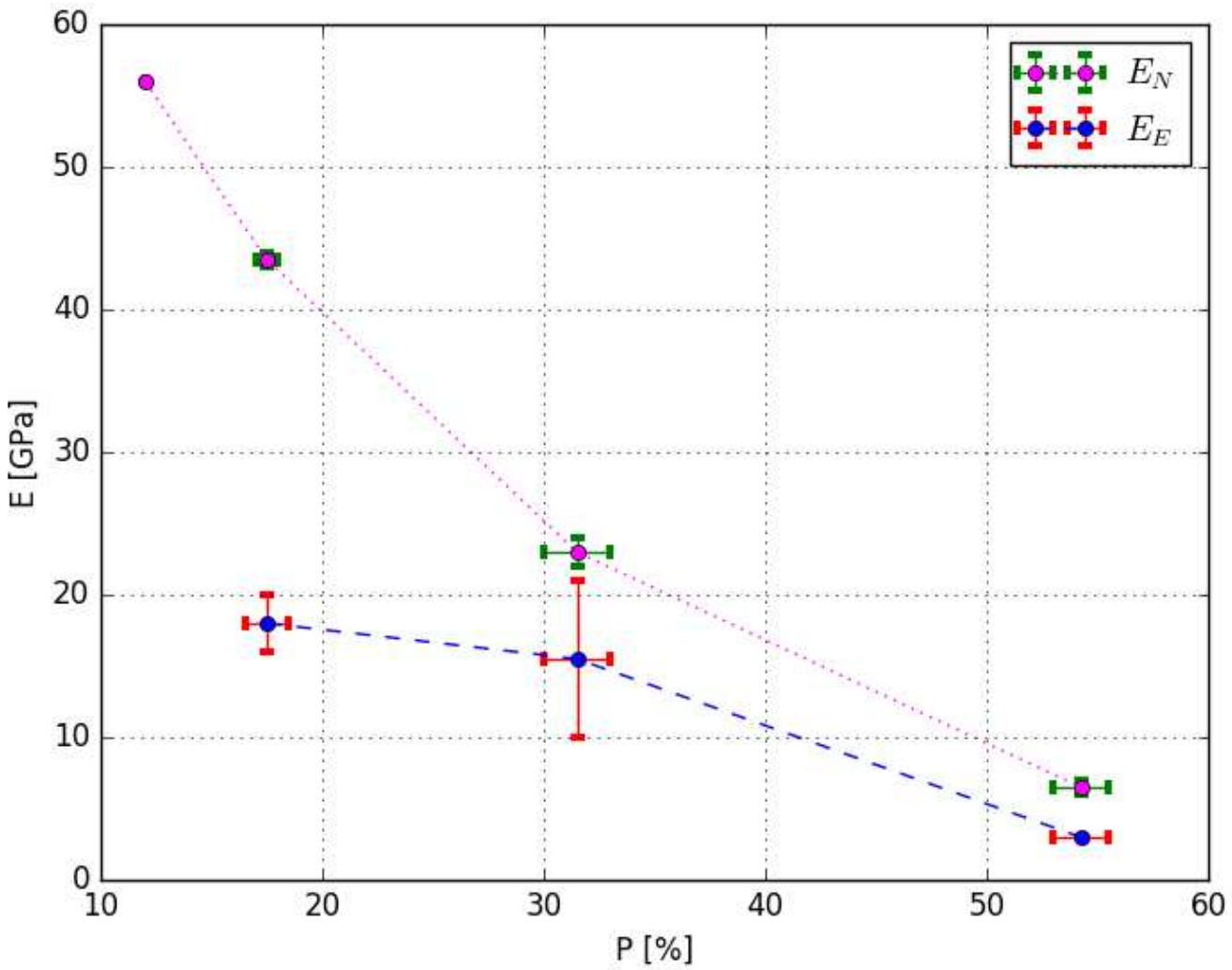

Figura 2: Variación del módulo de elasticidad $\mathrm{E}_{\mathrm{N}}$ y $\mathrm{E}_{\mathrm{E}}$ en función de la porosidad. 


\section{CONCLUSIÓN}

En este trabajo se planteó y logró bajar la temperatura de sinterizado de titanio poroso a valores que permitirían en un futuro, poder incorporar hidroxiapatita (HA) tal que este no se descomponga al reaccionar con el Ti.

- Se consiguieron muestras sinterizadas a partir de $\mathrm{TiH}_{2}$ con tratamientos térmicos realizados a 600 y $800{ }^{\circ} \mathrm{C}$.

- En base a los tiempos y temperaturas de sinterizado determinados se logró establecer un proceso para obtener gradientes de porosidad evitando la formación de interfaces que afecten a las propiedades mecánicas del mismo.

- Distintas pruebas realizadas permitieron obtener muestras con porosidad longitudinal y radial donde el estudio metalográfico reveló continuidad estructural entre capas.

- El modulo elástico de las muestras con porosidad radial se encuentra dentro del rango del módulo elástico del hueso.

\section{BIBLIOGRAFÍA}

[1] DUFF, G., Biomateriales. In: Materiales y materias primas (Capítulo 8). Instituto Nacional de Educación Tecnológica, Ministerio de Educación, República Argentina., 2011.

[2] COLLINS, E.W., The physical Metallurgy of Titanium Alloy, 2 ed., Ohio, ASM, Metals Park, 1984.

[3] OLDANI, C., LÓPEZ PADILLA, R., "Titanio poroso para implantes óseos", In: anales VI Congreso Latinoamericano de ingeniería biomédica, pp 261-264, Entre Ríos, Argentina, Oct. 2014.

[4] LOPEZ PADILLA, R., Desarrollo de estructuras porosas de titanio biocompatible, obtenidas por pulvimetalurgia, Tesis D. Sc., FCEFyN, UNC, Córdoba, Argentina, 2014.

[5] BLACK, J. "Titanium And Titanium Alloys". In: Black, J., Hastings, G. (eds). Handbook of Biomaterials Properties. Digital edition, chapter 16, London. Springer - Verlag. 1998.

[6] AMIETTA, J., BOEZIO, L., .Diseño e implementación de modelo experimental in vivo para evaluar titanio como material de reemplazo óseo, Tesis de grado, FCEFyN, UNC, Córdoba, Argentina, 2015.

[7] TORRES, Y., TRUEBA, P., PAVÓN, J., et al., "Designing, processing and characterization of titanium cilindres with graded porosity: an alternative to stress-shielding", Solutions Materials and Design. v 110, n. 63, pp. 316-324, 2014.

[8] LINDEN, S.S., M., QUADROS, A., M., SANTOS NETO, C.J., et al., "Biocompatibility of titanium implants with and without hydroxyapatite coating ; Biocompatibilida de entre implantes de titânio com e sem cobertura de hidroxiapatita", Revista da Faculdad de Odontologia -UPF, v. 1, n. 1., Brazil, 1996.

[9] APARICIO BÁDENAS, C., Tratamientos de superficie sobre titanio comercialmente puro para la mejora de la osteointegración de los implantes dentales,. Tesis de D, Sc., Universitat Politècnica de Catalunya. Departament de Ciència dels Materials i Enginyeria Metal-lúrgica, España, 2005.

[10] PAZ, A., MARTÍN, Y., PAZOS, L. M., et al., "Obtención de recubrimientos de hidroxiapatita sobre titanio mediante el método biomimético", Revista de Metalurgia, v 47, n. 2, pp. 138-145, 2011.

[11] CIPRIANO RANGEL, R., Efeito da implantação iônica por imersão em plasmas sobre a bioatividade de titânio, Tesis D.Sc., Universidade Estadual Paulista Júlio de Mesquita Filho (unesp) - Brasil, 2012.

[12] CANAVOSIO, L., GALlO, M., Sinterizado de titanio con Hidroxiapatita, Tesis de grado. FCEFyN. UNC. Córdoba, Argentina, 2010.

[13] YANG, Y., KIM, K.-H., AGRAWAL, C. M., ONG, J. L., "Interaction of hydroxyapatite-titanium at elevated temperature in vacuum en- vironment", Biomaterials, v .25, n.15, pp. 2927-2932, 2014.

[14] MOMOSE, D.R., Desenvolvimento de interfaces com gradiente funcional para a sinterização simultânea do titânio/hidroxiapatita, Tesis D. Sc, Instituto Tecnológico de Aeronáutica, Brazil, 2009.

[15] MARCELO, T. M., LIVRAMENTO, V., OLIVEIRA, M. V. D., et al., "Microstructural characterization and interactions in Ti-and $\mathrm{TiH}_{2}$-hydroxyapatite vacuum sintered composites", Materials Re- search. v. 9, n. 1, pp. 65-71, 2006.

[16] BALBINOTTI, P., Elaboração e caracterização de compósitos titânio/hidroxiapatita por metalurgia do pó para aplicações biomédicas. Tesis D. Sc., Universidade do Estado de Santa Catarina - UDESC, Brazil, 2011. 
[17] MILNE, R., Recuperación pulvimetalúrgica de aleación de magnesio AZ91D con fines estructurales, Tesis de grado, FCEFyN, UNC, Córdoba, Argentina. 2015

[18] NIELSEN, L.F., CERAM. J.,"Elasticity and Damping of Porous Materials and Impregnated Materials", Journal of the American Ceramics Society, v.67, n. 2, pp. 93-98, 1984.

[19] TORRES, Y., LASCANO, S., BRIS, J., PAVÓN, J., et al., "Development of porous titanium for biomedical applications: A comparison between loose sintering and space-holder techniques”, Materials Science and Engineering: C, v 37, pp. 148-155, 2014. 